\title{
Samarium-153 ethylenediamine tetramethylene phosphonate therapy for bone pain palliation in skeletal metastases
}

\author{
Tripathi M, Singhal T, Chandrasekhar N, Kumar $\mathbf{P}^{\star}$, Bal C, Jhulka $\mathrm{PK}^{\star *}$, \\ Bandopadhyaya G, Malhotra A \\ Departments of Nuclear Medicine, ${ }^{*}$ Biostatistics and ${ }^{* *}$ Radiotherapy, All India Institute of Medical \\ Sciences, New Delhi, India
}

Correspondence to: Dr. Madhavi Tripathi, E-mail: madhu_deven@yahoo.com

\section{Abstract}

BACKGROUND: Systemic therapy with radionuclides may be used for the treatment of patients with painful skeletal metastases owing to its efficacy, low cost and low toxicity. Imported radionuclides for pain palliation, like Strontium89 are expensive; particularly for developing countries. In the Indian scenario, Samarium-153 (Sm-153) is produced in our own reactors and as a result, it is readily available and economical. AIM: We undertook this study to determine the efficacy and toxicity of single-dose Sm-153 ethylenediamine tetramethylene phosphonate as a palliative treatment for painful skeletal metastases. MATERIALS AND METHODS: Eightysix patients with painful skeletal metastases from various primaries, were treated with Sm-153 EDTMP at a dose of $37 \mathrm{MBq} / \mathrm{kg}$. The effects were evaluated according to change in visual analogue pain score, analgesic consumption, Karnofsky performance score, mobility score and blood count tests, conducted regularly for 16 weeks. STATISTICS: Repeated measures analysis. RESULTS: The overall response rates were $73 \%$, while complete response was seen in $12.4 \%$. Reduction in analgesic consumption with improvement in Karnofsky performance score and mobility score, was seen in all responders. Response rates were 80.3 and $\mathbf{8 0 . 5 \%}$ in breast and prostate cancer, respectively. One case, each of Wilms tumor, ovarian cancer, germ cell tumor testis, multiple myeloma, primitive neuroectodermal tumor and oesophageal cancer, did not respond to therapy. No serious side-effects were noted, except for fall in white blood cell, platelet and haemoglobin counts, which gradually returned to normal levels by six-eight weeks. CONCLUSION: Sm-153 EDTMP provided effective palliation in $73 \%$ patients with painful bone metastases: the major toxicity was temporary myelosuppression.

Key words: Sm-153 EDTMP, bone pain, palliation, myelotoxicity, strontium radioisotopes, therapeutic use, bone neoplasms, secondary, palliative care, humans.

Skeletal metastases are present in the majority of patients with end-stage malignancies. The therapeutic options are rarely (if ever) curative and at some point of time the vast majority of patients suffering from osseous metastasis will develop progressive disease, leading to a series of disease related events that have the most significant impact on the quality of life in these patients. Non-opioid analgesics are usually the first-line treatment and can provide adequate pain relief initially, however as the disease progresses, increased dose of opioid analgesics are required and this is frequently associated with increased adverse effects, including constipation and drowsiness. Chemotherapy or hormonal therapy may be used for both soft tissue and bone metastases and can be effective until the disease becomes refractory to these agents. External beam radiotherapy provides effective pain control with short courses of high dose per 
fraction and a low toxicity, if the metastatic disease is not extensive; however the toxicity rapidly increases with wide radiation fields. ${ }^{[1]}$ Systemic therapy with radionuclides linked to bone seeking agents, is a treatment option for patients with disseminated skeletal metastases, owing to its efficacy, low cost and low toxicity. ${ }^{[2]}$ Radionuclides suitable for systemic metabolic radiotherapy of bone pain include Phosphorous-32 (P-32), Strontium-89 (Sr-89), Rhenium-186 (Re-186) chelated with hydroxyethylidene diphosphonate (HEDP) and Samariumm-153 (Sm-153) chelated with ethylene diamine tetramethylene phosphonate (EDTMP). ${ }^{[3-5]}$ Considerable bone marrow suppression due to the presence of higher energy $\beta$ particles, is the major constraint towards the widespread use of P-32 (Mean $\beta=695 \mathrm{keV}, \mathrm{tl} / 2=14.3$ days) and $\mathrm{Sr}-89$ (Mean $\beta=583 \mathrm{keV}, \mathrm{tl} / 2=50.5$ days). Apart from that, the absence of image-able $\gamma$ photons and long half life (especially in case of Sr-89), are often cited as drawbacks. Beta emitters with short half-lives, like Re-186 (Mean $\beta=362 \mathrm{keV}, \gamma=137 \mathrm{keV}, \mathrm{tl} / 2=3.7$ days) and $S \mathrm{~m}-153$ (Mean $\beta=233 \mathrm{keV}, \gamma=103 \mathrm{keV}, \mathrm{tl} / 2=1.9$ days), deliver their radiation dose at higher dose rates, which may be therapeutically more effective than equivalent doses given at lower dose rates. The short range of beta emission of these radionuclides may be of advantage in limiting red marrow irradiation. Sm-153-EDTMP localizes in the skeleton by chemi-absorption of the tetraphosphonate by hydroxyapatite and by the formation of Samarium oxide involving an oxygen on the hydroxyapatite molecule. Early phase I/II studies were published more than ten years $\operatorname{ago}^{[6-9]}$ and since then, this agent has been clinically used world wide for pain palliation in symptomatic bone metastases from several cancers, mainly prostate and breast. In the Indian scenario, $\mathrm{Sm}-153$ is produced in our own reactors and thus it is readily available and more economical than other agents like Sr-89, which have to be imported. We undertook this prospective study to investigate the efficacy and toxicity of Sm-153-EDTMP in the palliative treatment of painful skeletal metastases.

\section{Materials and Methods}

From April 2001 to September 2004, 110 patients were enrolled in the study, of whom 86 patients completed the 16-week follow-up. Age range of patients was 17 to 80 years (mean $=53.2$ years). There were 41 males and 45 females. Primary tumors included, were breast $=46$, prostate $=31$, lung $=3$ and one case each of germ cell tumor testis, ovarian carcinoma, oesophageal cancer, primitive neuroectodermal tumor, wilms tumor and multiple myeloma.
All patients underwent whole body Tc-99m methylenediphosphonate (MDP) bone scan, relevant blood counts and liver and kidney function tests, prior to inclusion in the study. Inclusion criteria for the patients were: two or more sites of painful bone metastases corresponding to positive sites on bone scintigraphy, progressive pain or pain requiring increasing level of analgesics, Karnofsky performance score of $\geq 60$, recurrent pain in a radiotherapy field, normal renal function, normal liver function, platelet count greater than $150 \times 10^{9} / 1$, leucocyte count greater than $4000 / \mathrm{cu} . \mathrm{mm}$, more than a month post myelosuppressive chemotherapy and a gap of atleast one week if bisphosphonate therapy had been given before Sm-153 therapy. Patients were excluded if they had extensive soft tissue metastases and less than three months projected survival.

The study protocol was approved by the Medical Ethics Committee of the Institute. Following inclusion in the study, each patient was explained the details of the procedure, benefits and side effects of therapy and the follow-up protocol. 45 patients were on non-narcotic analgesics, 36 patients were on codeine and five patients were on oral morphine. A majority of patients had undergone single fraction high dose radiotherapy, to one or multiple sites. 14 patients had been treated with bisphosphonates (six to eight doses). A written consent was taken prior to dose administration. Patients were then instructed to keep taking the analgesics and hormonal therapy, as prescribed to them. Pain was evaluated using a patient-rated pain intensity visual analogue scale (VAS), where the patient was asked to score pain on a 10 -step scale, 0 being nearly no pain and 10, the maximal pain. An average weekly pain score was derived from the daily pain score. For each patient, an analgesic score was computed as the product of analgesic type and administration frequency coded into integer form. Type: $0=$ none; $1=$ non-narcotic; $2=$ mild oral narcotics like codeine $<30 \mathrm{mg} ; 3=$ moderate oral narcotics, $30-60 \mathrm{mg}$ codeine or equivalent; $4=$ parenteral narcotics or sustained release morphine. Frequency of administration: $0=$ none; $1=$ as needed but not daily; $2=$ one tablet per day; $3=$ one to four tablets per day; $4=$ more than four tablets per day; $5=$ parenteral narcotics. Mobility assessment included the following: a) ability to sit and rise from a chair, b) ability to dress/undress, c) ability to wash oneself, d) ability to work at home and e) ability to travel outside the house. Each of these abilities had a score assigned to them, as follows: $0=$ unable; $1=$ able with assistance; $2=$ able unaided with some difficulty; $3=$ normal activity. The scores for each item were totalled and averaged to get a single mobility score, in the range zero to three. The patient was asked to 
record the pain score and analgesic intake from the time he took the appointment, or atleast 14 days prior to therapy, so that he could be familiarised to the technique.

Bone disease was assessed by Tc-99m MDP bone scanning, with assignment of a five-point scintigraphic score, based on the number or extent of metastases, according to the criteria of Soloway et al. ${ }^{[10]}$

Sm-153 EDTMP was obtained from Bhabha Atomic Research Centre, a division of the Department of Atomic Energy, Mumbai. Sm-153 is produced by neutron activation of natural Samarium in the nuclear reactor. Complexation of Sm-153 with EDTMP is carried out by addition of alkaline EDTMP, mixing and adjusting $\mathrm{pH}$ to $7-8$ and heating over a water bath at $60-70^{\circ} \mathrm{C}$. The entire procedure is carried out under aseptic conditions. Radiochemical purity of $>98 \%$ is obtained.

Patients were administered the dose on an outpatient basis. $37 \mathrm{MBq} / \mathrm{kg}$ body weight of Sm-153 EDTMP was given by slow intravenous injection. This was followed by a whole body scan after two hours, on a dual head gamma camera (Millenium VG, GE, HaifaIsrael), using a $20 \%$ window centered at $103 \mathrm{keV}$. The patient was explained the follow up protocol and was allowed to return home. No other tumor-oriented therapy was allowed upto 16 weeks post therapy.

All patients were followed up for 16 weeks, posttherapy. Analgesic consumption, symptoms and sleeping patterns were recorded by the patients and/or family members. The patient was allowed to adjust the dose of his/her medications if symptoms changed. The patients were asked to get the haemogram done weekly upto 12 weeks and provide the report to the physician telephonically. At the end of four weeks, the patient was to come to the clinic for the first detailed assessment and recording of data. The VAS pain score, analgesic score, mobility index and Karnofsky performance scores were recorded at four, eight, 12 and 16 weeks, taking the mean of weekly scores. Each patient was closely followed for onset of pain relief and duration of response. The following variables were also monitored with respect to primaries; Serum alkaline phosphatase, Prostate specific antigen (PSA) and CEA/CA15.3. A bone scan with Tc-99m MDP was repeated at 16 weeks.

Pain palliation: For primary data analysis, the end points of the study were the VAS results and analgesic consumption. If the pain intensity on the VAS decreased for atleast two steps for two weeks, while the analgesic score remained atleast constant; therapy with Sm-153 was considered as efficacious and the patient was a responder. If the patient was completely relieved of pain, a complete response was recorded. Parameters evaluated, included onset of therapeutic response and duration of response. If the pain score or analgesic score increased during the follow up period, the patient was considered a non responder.

Toxicity was evaluated by measuring changes in platelets, leucocytes and haemoglobin, during the first three months of follow-up and were classified by the five-point toxicity score according to World Health Organization criteria. Patients were also evaluated for a possible pain flare (flare phenomenon), beginning at 4872 hours after the therapy and lasting for two to three days. This would predict a reduction in bone pain i.e., a therapeutic response.

All numeric data was expressed as mean \pm SD. For each patient, post-treatment data was compared with pre-treatment data (baseline). Because the scores fluctuated considerably over the week, mean scores for VAS, analgesic scores and Karnofsky and mobility scores, were calculated at four, eight, 12 and 16 weeks. Repeated measures analysis was then used to calculate the statistical significance of pre-therapy and interval changes in post-therapy scores. A $P$ value of $<0.05$ was considered as significant. Chi square test was used for comparison of response between carcinoma $(\mathrm{Ca})$ breast and $\mathrm{Ca}$ prostate patients. To test for significance of pretherapy difference in age, VAS and analgesic score between responders and non-responders, Unpaired $t$ test was applied.

\section{Results}

A total of 63 patients responded to single dose therapy with Sm-153 EDTMP and 23 patients were nonresponders. The therapeutic effect started at four to 14 days after administration, with an average of $8.8 \pm 5.3$ days. The response was mild initially, then increased to its full extent, during which many patients could decrease their analgesic consumption. The effect lasted for two to eight months; and the mean duration of the response was 3.16 \pm 1.88 months. Approximately $12.4 \%$ (11/86) of patients were completely pain-free for $5.18+1.7$ months (range 3 to 8 months), post-therapy. The responders showed an improvement in general condition and a better quality of life. A good correlation was found between the Tc-99m MDP images before therapy and the Sm-153 images obtained 24-hr after therapy. [Figure 1]

A total of $46 \mathrm{Ca}$ breast patients with painful bone 


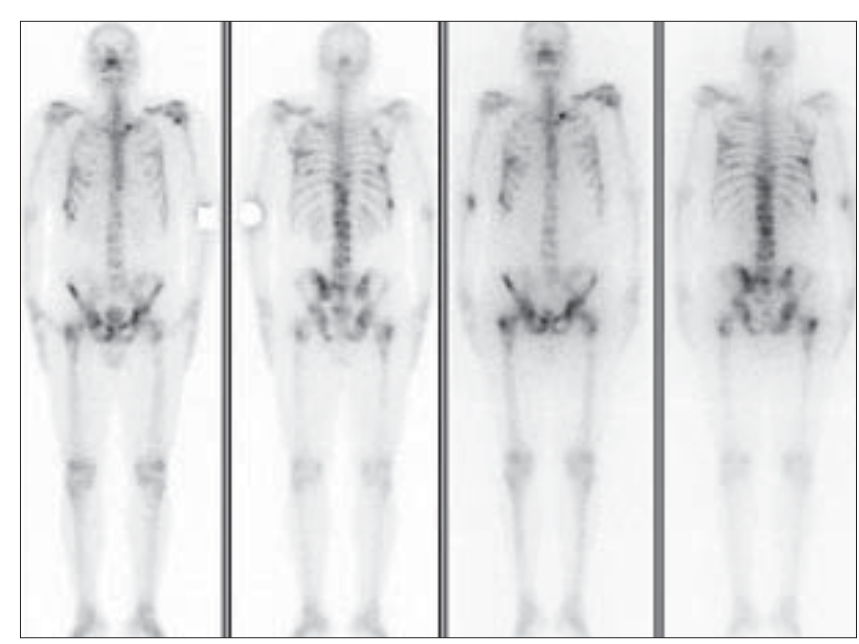

Figure 1: Tc-99m MDP bone scan, anterior and posterior views and the Sm-153 post-therapy scan, anterior and posterior views of a male cancer breast patient with multiple skeletal metastasis

metastases were included for therapy. This included 44 females and two males. Age was $50 \pm 12$ years (range 32 to 80 years). The response rate in Ca breast patients was $80.4 \%(37 / 46)$ [Table 1]. Statistically significant reduction in pain scores and analgesic scores from the baseline were seen at four, eight, 12 and 16 weeks, post-therapy $(P<0.001,95 \%$ confidence interval) [Table $2]$. Six patients had complete response to therapy (complete absence of pain) for $5.67 \pm 1.63$ months and were not on any analgesics during this duration. Subsequently, two of these patients received a second dose and three patients received three doses of Sm-153. Nine patients did not respond to therapy, with an increase in pain score and analgesic index [Table 3]. Four of these patients were on oral Morphine pretherapy and had to continue Morphine. Two patients were on oral narcotics (Codeine) prior to therapy and one of them had to be started on Morphine during the follow up period. This patient had a pain flare in the

Table 1: Therapeutic response to Sm-153 EDTMP therapy.

\section{Ca breast Ca prostate}

\begin{tabular}{lcc}
\hline Response onset (days) & & \\
\hline Mean $\pm S D$ & $8.7 \pm 5.21$ & $8.6 \pm 5.40$ \\
\hline Range & 6 to 14 & 4 to 10 \\
\hline Duration (months) & & \\
\hline Mean $\pm S D$ & $3.2 \pm 1.95$ & $3.1 \pm 1.82$ \\
\hline Range & 2 to 8 & 2 to 7 \\
\hline Complete response & $6 / 46$ & $5 / 31$ \\
\hline Partial response & $31 / 46$ & $20 / 31$ \\
\hline No response & $9 / 46$ & $6 / 31$ \\
\hline
\end{tabular}

Table 2: Pre-therapy and post-therapy VAS and analgesic scores in responders.

\begin{tabular}{|c|c|c|c|c|}
\hline \multirow[t]{2}{*}{ Responders } & \multicolumn{2}{|c|}{ Ca breast } & \multicolumn{2}{|c|}{ Ca prostate } \\
\hline & VAS & $\begin{array}{l}\text { Analgesic } \\
\text { score }\end{array}$ & VAS & $\begin{array}{l}\text { Analgesic } \\
\text { score }\end{array}$ \\
\hline Pre-therapy & $7.52 \pm 1.27$ & $6.63 \pm 3.92$ & $7.48 \pm 1.41$ & $4.84 \pm 3.09$ \\
\hline 4 wks & $3.86 \pm 1.49^{*}$ & $4.96 \pm 3.52 *$ & $4.90 \pm 1.90 *$ & $3.60 \pm 2.76^{*}$ \\
\hline 8 wks & $1.78 \pm 1.11^{*}$ & $3.45 \pm 3.45^{\star}$ & $2.20 \pm 1.59 *$ & $2.66 \pm 2.94^{*}$ \\
\hline 12 wks & $1.85 \pm 1.18^{*}$ & $3.45 \pm 3.44^{\star}$ & $2.33 \pm 1.59 *$ & $2.66 \pm 2.94$ * \\
\hline 16 wks & $1.92 \pm 1.25^{*}$ & $3.66 \pm 3.60 *$ & $2.51 \pm 1.61^{*}$ & $2.73 \pm 2.98$ * \\
\hline \multicolumn{5}{|c|}{ *Indicates $P$ value of $<0.001$ at $95 \%$ confidence interval. } \\
\hline \multicolumn{5}{|c|}{$\begin{array}{l}\text { Table 3: VAS pain score and analgesic scores in } \\
\text { non-responders. }\end{array}$} \\
\hline \multirow{2}{*}{$\begin{array}{l}\text { Non- } \\
\text { responders }\end{array}$} & \multicolumn{2}{|c|}{ Ca breast } & \multicolumn{2}{|c|}{ Ca prostate } \\
\hline & VAS & $\begin{array}{l}\text { Analgesic } \\
\text { score }\end{array}$ & VAS & $\begin{array}{l}\text { Analgesic } \\
\text { score }\end{array}$ \\
\hline Pre-therapy & $7.88 \pm 1.22$ & $10.11 \pm 3.85$ & $7.16 \pm 0.75$ & $10.66 \pm 4.36$ \\
\hline 16 wks & $8.55 \pm 1.01$ & $13.55 \pm 3.08$ & $7.66 \pm 0.81$ & $13.33 \pm 2.06$ \\
\hline
\end{tabular}

first week, post-therapy. One of the patient on nonnarcotic analgesics, had to be started on codeine, while the remaining two had to increase the dose of nonnarcotic analgesics being taken for pain relief.

The Karnofsky scale which was used in an attempt to quantify the improvement in the patient's condition after the treatment, improved in all responders (mean pre-therapy $s c o r e=70.83 \pm 8.74$ and 16 week score $=79.16 \pm 9.06)$. Generally, improved mobility accompanied an improvement in pain scores (mean pretherapy score $=1.72 \pm 0.38$ and 16 week score $=2.14 \pm 0.47$ ).

A total of 31 hormone-resistant $\mathrm{Ca}$ prostate patients age $63 \pm 8.3$ years (range 50 to 80 years), were administered $\mathrm{Sm}-153$ therapy. The response rate in Ca prostate patients was $80.6 \%(25 / 31)$, [Table 1]. Statistically significant reduction in pain scores and analgesic scores from the baseline, were seen at four, eight, 12 and 16 weeks, post-therapy $(P<0.001,95 \%$ confidence interval), [Table 2]. Five patients had complete response to therapy for $4.6 \pm 1.8$ months. Subsequently, two of these patients received a second dose and three patients received three doses of $S m-153$. Six patients did not respond to therapy, with an increase in pain scores and analgesic index, at the end of the evaluation period [Table 3]. Three patients on oral codeine had to increase the dose of codeine, for pain relief. One patient on oral codeine had to be started on oral morphine and 
the patient taking oral morphine pre-therapy, had to continue morphine at an increased dose. One patient on non-narcotic analgesics had to be started on codeine.

The Karnofsky scale, which was used in an attempt to quantify the improvement in the patient's condition after the treatment, improved in all responders (mean pre-therapy score $=74 \pm 7.07$ and 16 week score $=83.2 \pm 7.48)$. Generally, improved mobility accompanied an improvement in pain scores (mean pretherapy $s c o r e=1.88 \pm 0.21$ and 16 week score $=2.23 \pm 0.48)$.

The median bone scan index pre-therapy for both $\mathrm{Ca}$ breat and Ca prostate patients, was two. The metastatic pattern on the bone scan remained essentially unchanged in most cases post-therapy, with a median bone scan index post-therapy, of two. No change in alkaline phosphatase or sPSA levels could be documented in $\mathrm{Ca}$ breast and $\mathrm{Ca}$ prostate patients, respectively, at the end of 16 weeks.

There was significant difference between pre-therapy analgesic scores amongst responders and non-responders in Ca breast (mean pre-therapy score $=6.6 \pm 3.9$ in responders and $10.1 \pm 3.8$ in non-responders, $P=0.022$ ), while age and VAS did not reveal significant difference. Similarly, a significant difference between pre-therapy analgesic scores amongst responders and non-responders was seen in Ca prostate patients (mean pre-therapy score $=4.8 \pm 3.3$ in responders and 10.6 \pm 4.4 in nonresponders, $P=0.001$ ), while age and VAS did not reveal significant difference.

A $2^{*} 2$ table for responders and non-responders in $\mathrm{Ca}$ breast and Ca prostate patients revealed $\chi^{2}=0.001$ and $P=0.98$ (95\% confidence interval), thus indicating a similarity of response rates in both the groups.

Only one patient with non small cell lung carcinoma responded to therapy. He was a 40 year male on oral non-narcotic analgesics, thrice daily for pain relief. Following therapy, he had pain relief starting on the seventh day, following which, he decreased analgesics to one daily, or as required and relief lasted for three months. Karnofsky and mobility scores were stable, pre and post therapy. The other two patients (small cell $\mathrm{Ca}$ ) did not respond to therapy and they continued on oral codeine for pain relief, with dose increase in one patient. One case each of ovarian carcinoma, germ cell tumor testis, cancer oesophagus, primitive neuroectodermal tumor, wilms tumor and multiple myeloma, did not respond to therapy. Their pain scores increased progressively and at the end of the four month evaluation period, they were on oral morphine therapy for pain relief.

Five patients had leucopenia of grade I and 3 patients had grade II toxicity. Three patients each, showed grade I and Grade II thrombocytopenia. Eighteen patients had grade I, and eight patients had grade II anaemia. Grade III and IV haematological toxicity was not seen in any patient. In no case did bleeding occur and no patient needed transfusion. The results are summarized in Table 4. Haemoglobin, platelets and WBC decreased by approximately 13,23 and $26 \%$, respectively from baseline values, with nadir at 3-5 weeks and recovery by six to eight weeks.

No patient showed any clinically evident acute adverse side-effects following radionuclide administration and only one patient experienced a mild increase in pain i.e. a flare response, though this was not associated with a clinical response to therapy. No alterations in serum creatinine or liver enzymes levels could be documented.

\section{Discussion}

Sm-153 EDTMP, a recently recommended radiopharmaceutical to control skeletal pain, has relatively ideal physical, chemical and biological properties. It has been proved that the tracer is cleared from the blood quickly, with more than half the injected dose being deposited in bone and very little accumulation elsewhere. ${ }^{[11]}$ The $103 \mathrm{keV}$ gamma ray is suitable for imaging its in-vivo distribution. Many authors have reported favourable clinical experience with Sm-153 EDTMP and benefit has been documented in several multicentric trials. ${ }^{[12,13]}$ However, there have been no reports on the use of Sm-153-EDTMP in Indian patients, especially since this radiopharmaceutical is made in our reactors and is available to us at an economical price, compared to imported radionuclides like Sr-89. It was therefore of interest to investigate the response among Indian patients to this therapy, utilizing the standardized evaluation protocols.

In the present study, overall response rate was $73 \%$.

\begin{tabular}{|c|c|c|c|c|c|c|}
\hline \multirow[t]{2}{*}{ Diagnosis } & \multicolumn{2}{|c|}{ Leucopenia } & \multicolumn{2}{|c|}{ Thrombocytopenia } & \multicolumn{2}{|c|}{ Anemia } \\
\hline & $\begin{array}{c}\text { Grade } \\
\text { I }\end{array}$ & $\underset{\text { II }}{\text { Grade }}$ & $\begin{array}{c}\text { Grade } \\
\text { I }\end{array}$ & $\begin{array}{c}\text { Grade } \\
\text { II }\end{array}$ & $\underset{\mathrm{I}}{\text { Grade }}$ & $\begin{array}{c}\text { Grade } \\
\text { II }\end{array}$ \\
\hline Ca breast & $2 / 46$ & $1 / 46$ & $1 / 46$ & $1 / 46$ & $12 / 46$ & $4 / 46$ \\
\hline Ca prostate & $1 / 31$ & $2 / 31$ & $1 / 31$ & $2 / 31$ & $4 / 31$ & $2 / 31$ \\
\hline Ca lung & $1 / 3$ & 0 & 0 & 0 & $1 / 3$ & $1 / 3$ \\
\hline Others & $1 / 6$ & 0 & $1 / 6$ & 0 & $1 / 6$ & $1 / 6$ \\
\hline
\end{tabular}


This was comparable to the rates reported by the controlled clinical trials ${ }^{[12-14]}$ and the other uncontrolled studies reported in literature, ${ }^{[17-19]}$ where response rates varied from 61 to $95 \%$. The dose of Sm-153 used in these studies ranged from 18.5 to $111 \mathrm{MBq} / \mathrm{kg}$. A lower response was claimed to occur at lower doses, while there was no difference in the overall degree of pain palliation at doses ranging from 37 to $111 \mathrm{MBq} /$ $\mathrm{kg}$. Myelotoxicity however, was clearly dose-dependent. We used a standard fixed dose of $37 \mathrm{MBq} / \mathrm{kg}$, as it has a better therapeutic ratio than a dose of 18.5 $\mathrm{MBq} / \mathrm{kg}$ and a clear superiority of effect and safety of this dose was confirmed in the first two multicentric clinical studies, assessing the efficacy of Sm-153 EDTMP. ${ }^{[12,13]}$ Pain relief was noted as early as four days after dose administration, while the effect lasted for two to eight months. This was similar to that reported by the previous studies, where response duration varied from two to 35 weeks and response onset was as early as two days to as long as 14 days, after therapy. The short physical half-life of Sm-153 (1.9 days) offers the benefit of delivering a high dose rate over a short period of time, resulting in a greater biological effect and possibly explaining the early onset of pain relief.

The composition of our patient group was similar to that of others and included patients of $\mathrm{Ca}$ breast (41/ 86), Ca prostate (36/86), Ca lung (3/86) and six other primaries. The proportion of patients with $\mathrm{Ca}$ breast, however exceeded the other primaries, while the number of Ca lung cases was small unlike in other studies. ${ }^{[12-17]}$ The daily documented VAS and analgesic scores were reliable methods of assessing efficacy of therapy. Complete responders who received non-opiate analgesics before therapy with $S \mathrm{~m}-153$, could discontinue medication for upto 16 weeks, post-therapy. This indicates that Sm-153 had a palliative effect at an early stage of metastatic disease. On the other hand, patients on oral Morphine (five cases) prior to therapy, had to continue Morphine for pain relief and did not respond to therapy. It is possible that these patients had developed opiod dependence, due to long term use of opiod analgesics. This supports the need for intravenous radiopharmaceutical therapy, at an early stage of the bone disease. Palliative treatment with Sm-153 even resulted in pain reduction, if the patients experienced pain in regions where local radiation therapy had been applied before. Karnofsky performance score and mobility index were used as additional criteria for assessing response post therapy and showed an improvement in most responders.

After injection, response was recognized in $80.4 \%$ breast cancer and in $80.6 \%$ prostate cancer patients.
This is again comparable to the reports in literature, 40 to $85.5 \%$ response rates in $\mathrm{Ca}$ breast ${ }^{[6,12,18]}$ and 70 to $80 \%$ response in Ca prostate. ${ }^{[1]}$ Because Sm-153 reacts like diagnostically radiolabelled diphosphonates, it probably accumulates with preference in osteoblastic bone metastases. Osteoblastic metastases are found regularly in patients with metastases originating from prostate cancer. ${ }^{[19]}$ Because breast cancer is known to generate bone metastases of the osteoblastic and osteolytic types, it may be supposed that in these patients, the osteoblastic component of the metastases is highly responsible for the bone pain. Response rates and therapeutic effect was similar in both Ca breast and $\mathrm{Ca}$ prostate patients. Analgesic scores were slightly higher in the Ca breast patients, as most of them had been extensively treated (chemotherapy, hormonal therapy, radiotherapy and bisphosphonates) for bone metastases prior to referral for Sm-153 therapy, while most Ca prostate patients were referred directly for Sm153 therapy, following failure of hormonal manipulation. Interestingly, the two male Ca breast patients included for therapy with Sm-153, had complete response lasting for seven and eight months each. Whether such a good response to Sm-153 therapy in male $\mathrm{Ca}$ breast can be related to the biological difference of male breast $\mathrm{Ca}$ from female breast $\mathrm{Ca}$, needs further study, by including a larger number of patients. A total of $15 \mathrm{Ca}$ breast and $\mathrm{Ca}$ prostate patients did not respond to therapy. Though age and VAS pre-therapy was not significantly different between responders and non-responders, a significant differerence was noted in the analgesic scores pre-therapy, between the two groups. Analgesic scores, being higher in nonresponders, this again suggests the need for palliative radiopharmaceutical therapy at an early stage of the bone disease.

In their study Tian et al, ${ }^{[16]}$ demonstrated a good response to Sm-153 therapy of oesophageal cancers with bone metastases, while the group that did not respond to therapy, included a disproportionately larger number of patients with Cancer lung. In our study, only one of the three lung cancer patients responded to therapy, while none of the patients in the other primaries group responded to therapy. This confirms the observation of others, that the type of primary disease may have an effect on the pattern of metastases ${ }^{[9,20]}$ and as a consequence, influences the response to Sm-153 EDTMP. However, in view of the limited number of cases, it is still too early for conclusions to be drawn in this regard.

No serious side effects were encountered in any patient. Only one Ca breast patient complained of a pain flare in the first week post-therapy; this however was not followed by a clinical response and the patient had to 
be started on oral morphine. A flare response has been demonstrated to occur in seven to $10 \%,{ }^{[13,21]}$ of patients undergoing Sm-153 therapy, but whether this is associated with a therapeutic response is questionable, since this phenomenon has been reported to occur in placebo treated patients and non-responders also. Temporary inhibition of marrow function, with reduction in the haemoglobin, WBC and platelet counts, was encountered in most patients, with a nadir at three to four weeks and recovery by six to eight weeks, indicating a reversibility of myelotoxicity. This was similar to the pattern reported in most other studies. ${ }^{[12-17]}$ Grade III or IV haematological toxicity was not seen in any patient. However, in variance to other studies, we found a higher incidence of grade I and II anaemia, which included 16 patients of $\mathrm{Ca}$ breast and six patients of $\mathrm{Ca}$ prostate. This was probably, because most patients of $\mathrm{Ca}$ breast entered the study with a low haemoglobin value due to prior rigorous chemotherapy and also in breast cancer patients, metastatic disease often involves the bone marrow. Both these factors lead to a decreased bone marrow reserve, which cannot always be diagnosed by simple blood count measurements. Except for the higher incidence of anaemia in breast cancer patients, there was no significant difference in the number of patients showing the various grades of toxicity among the various primaries.

Shortcomings of our study include the following: 1) dosimetry was not performed for the bone lesions; 2) the tumor burden of patients was not calculated and related to their response; 3 ) it was difficult to eradicate subjectivity in judging response, as is true for most such studies.

Many questions remain concerning Sm-153 EDTMP treatment, including whether the radiopharmaceutical possesses a tumoricidal effect and whether therapy influences patients long-term survival. Additional protocols extending the use of this agent either alone, or in combination with other agents like bisphosphonates, low dose chemotherapy or radiotherapy, need investigation.

\section{Conclusion}

The results of this study showed that Sm-153 EDTMP offers a safe and effective treatment option in Indian patients with painful bone metastases. It is a systemic, simple and well-tolerated single-session procedure that usually achieves good pain palliation and sometimes pain-free periods lasting several months.

\section{References}

1. Hoskin PJ. Radiotherapy for bone pain. Pain 1995;63:137-9.

2. Dearnaley DP, Bayly RJ, A'Hern RP, Gadd J, Zivanovic MM, Lewington VJ. Palliation of bone metastases in prostate cancer. Hemibody irradiation or strontium-89? Clin Oncol 1992;4:101-7.

3. Serafini AN. Current status of systemic intravenous radiopharmaceuticals for the treatment of painful metatstatic bone disease. Int J Radiat Oncol Biol Phys 1994;30:1187-94.

4. McEwan AJ. Unsealed source therapy of painful bone metastases:an update. Semin Nucl Med 1997;27:165-82.

5. Serafini AN. Therapy of metastatic bone pain. J Nucl Med 2001;42:895-906.

6. Turner JH, Claringbold PG, Hetherington EL, Sorby P, Martindale AA. A phase I study of samarium-153 ehylenediaminetetramethylene phosphonate therapy for disseminated skeletal metastases. J Clin Oncol 1989;7:1926-31.

7. Podoloff DA, Kasi LP, Kim EE, Fossella FV, Bhadkamar VA. Evaluation of Sm-153 EDTMP as a bone imaging agent during a therapeutic trial. J Nucl Med 1991;32:A918.

8. Eary JF, Collins C, Stabin M, Vernon C, Petersdorf S, Baker M, et al. Sm-153-EDTMP biodistribution and dosimetry estimation. J Nucl Med 1993;34:1031-6.

9. Turner JH, Claringbold PG. A phase II study of treatment of painful multifocal skeletal metastases with single and repeated dose of samarium- 153 ethylenediaminetetramethylene phosphonate. Eur J Cancer 1991;27:1084-6.

10. Soloway MS, Hardeman SW, Hickey D, Raymond J, Todd B, Soloway S, et al. Stratification of patients with metastatic prostate cancer based on extent of disease on initial bone scan. Cancer 1988;61:195-202.

11. Collins C, Eary JF, Donaldson G, Vernon C, Bush NE, Petersdorf $S$, et al. Samarium-153 EDTMP in bone metastases of hormone refractory prostate carcinoma. A phase I/II trial. J Nucl Med 1993;34:1839-44.

12. Resche I, Chatal JF, Pecking A, Ell P, Duchesne G, Rubens R, et al. A dose-controlled study of Sm-153 EDTMP in the treatment of patients with painful bone metastases. Eur J Cancer 1997;33: 1583-91.

13. Serafini AN, Houston SJ, Resch I, Quick DP, Grund FM, Ell PJ, et al. Palliation of pain associated with metastatic bone cancer using samarium-153 Lexidronam: A double-blind:a double blind placebo controlled clinicak trial. J Clin Oncol 1998;16:1574-81.

14. Tian JH, Zhang JM, Hou QT, Oyang OH, Wang JM, Luan ZS, et al. Multicentre trial on the efficacy and toxicity of single-dose samarium-153-ethylene diamine tetramethylene phosphonate as a palliative treatment for painful skeletal metastases in China. Eur J Nucl Med 1999;26:2-7.

15. Li L, Liang Z, Deng H, Kuang A, Tan T, Luo S. Samarium-153-EDTMP bone uptake rate and its relation to therapeutic effect. Chin Med J 2002;115:1096-8.

16. Dolezal J, Vizd'a J, Cermakova E. Myelotoxicity after systemic radionuclide therapy of painful bone metastases with 153Samarium-EDTMP. Vnitr Lek 2003;49:189-93.

17. Wu H, Tan T, Fang L, Zhang X. Evaluation of efficacy of Sm-153EDTMP in patients with painful bone metastases of breast cancer. Sichun Da Xue Bao Yi Xue Ban 2003;34:716-8.

18. Turner JH, Martindale AA, Sorby P, Hetherington EL, Fleay RF, Hoffman RF, et al. Samarium-153 EDTMP therapy of disseminated skeletal metastases. Eur J Nucl Med 1989;15:784-95.

19. Jacobs SC. Spread of prostatic cancer to bone. Urology 1983;1:337-40.

20. Mc Neil BJ. Value of bone scanning in neoplastic diseases. Semin Nucl Med 1984; 14:277-86.

21. Friedland J. Local and systemic radiation for palliation of metastatic disease. Urol Clin N Am 1999;26:391-402. 